\title{
Global Product Development: Organization and Links with the Supply Chain
}

\author{
Francesca Faggioli ${ }^{1}$, Riccardo Franzini ${ }^{1}$, Margherita Pero ${ }^{1}$, \\ Monica Rossi ${ }^{1}$, and Sergio Terzi ${ }^{2}$ \\ ${ }^{1}$ Politecnico di Milano, Department of Management, Economics and Industrial Engineering \\ Via Lambruschini 4/b 20156, Milano, Italy \\ \{francesca.faggioli, riccardo.franzini\}@mail.polimi.it, \\ \{margherita.pero,monica.rossi\}@polimi.it \\ ${ }^{2}$ Università degli studi di Bergamo, Marconi 5, 24044, Dalmine (BG), Italy \\ sergio.terzi@unibg.it
}

\begin{abstract}
The coordination of supply chain configuration with new product development process has become extremely relevant to either prevent product launch from failure or to enhance supply chain effectiveness. Recently, along with the offshoring of production activities, a new paradigm emerges: companies adopt globally dispersed teams for the collaborative development of new products. While many studies regarding new product development have been carried out, few have examined this topic in a global setting. The aim of this research is to investigate how companies are organizing and managing product development projects on a global scale. Seventeen companies working in the electro-mechanical sector participated in this exploratory study to determine what type of global product development configurations were being adopted and to identify the link between the configurations and the supply chain. In addition, the study also addressed how the selected configurations influence new product development practices and which contingent factors determine the adoption of a certain configuration.

From the data, it emerges that common behaviours were adopted concerning geographical dispersion and the management of development teams in new product development. Furthermore, the study led to some interesting implications for managers because the identified configurations can be utilized by firms for more effective planning of their global product development efforts.
\end{abstract}

Keywords: Global Product Development, Globalization of Supply Chain, Global teams.

\section{Introduction}

Nowadays, achieving success in an international context requires companies to develop new products and services that respond effectively to many complex and diverse market needs and characteristics. With the emergence of a global economy and the acceleration of technological dynamics, companies have not only to be able to manage globalized supply chains (SC) [1], but also to deploy a successful international new 
product development (NPD) program in order to gain a competitive edge in the market. Finally, companies should be able to coordinate supply chain management with NPD processes to either prevent product launch from failure or to enhance supply chain effectiveness [2].

Global product development (GPD) has been defined by Eppinger and Chitkara [3] as " a single, coordinated product development operation that includes distributed teams in more than one country utilizing a fully digital and connected, collaborative product development process. This may include third parties that provide engineering or design capacity, or it may be an entirely captive, company-owned operation”. Therefore, the new challenge for multinational companies is to achieve success in their GPD practice, and to be able to manage the relations of GPD with globalized supply chains. Despite the relevance of the topic for companies, this topic has been only marginally explored by existing literature, and a study that presents how companies manage GPD and the relations with the supply chain is missing. Thus the aim of this work is to investigate how companies are organizing their worldwide distributed product development activities, with a particular focus on the ownership of these functions, distinguishing whether these are owned by the company or by a third party. Moreover, we propose to study the existing link between the resulting configurations and the company's supply chain.

The remainder of the paper is organized as follows: first a literature review on GPD and the link between GPD and global supply chain is presented. Then the research questions and the methodology used to answer to them is described. The discussion of results follows. The paper ends with the conclusions, limitations and future research paths.

\section{Global Product Development: Literature Review}

The existing literature describes the new product development as "the set of activities that start from a market opportunity and end with the production, sale and distribution of the production of the product" [4]. Many generic models for NPD process have been proposed [4] [5]. Companies can involve many different actors during NPD process, ranging from suppliers to universities, with various degree of collaboration [6] [7].

Eppinger and Chitkara [3] find that in the first five years of the 21st century, companies were rapidly migrating from a centralized approach to GPD practices: they exploit highly distributed, networked development process, in which centralized functions work with resources located in other sites or regions of the world [3]. GPD practice results in establishing GPD teams comprised of individuals drawn from multiple countries and company functions [8]. This is happening in several industrial settings, ranging from computer hardware and software development to automotive development [9]. And, in the recent years, the use of GPD approach involving multiple companies that are geographically dispersed and separated by organizational boundaries (e.g., outsourcing, offshoring, alliance) to develop products has spread dramatically [10]. Globalization practices are for the most part adopted by companies in developed 
countries and to a lesser extent by companies in developing countries [11] [12] [13]. Moreover, firms of smaller countries have a greater need to offshore their development facilities due to the lack of available resources in their origin countries [14] [13].

Some attempts to describe the emerging organizational forms of NPD have been done. However, to our knowledge, the classifications proposed cannot capture the diversity in the GPD organizational forms being too generic. In particular, based on the concepts of outsourcing (i.e., the ownership of the resources involved in NPD activities) and offshoring (i.e., the location of the resources involved in NPD activities), Eppinger and Chitkara [3] suggest four fundamental organizational forms of NPD, i.e. centralized, local outsourcing, captive offshore and global outsourcing. Besides, PTC [15] defines a GPD maturity model composed by five levels, from a totally centralized solution, to a globally dispersed one. GPD teams assume very high importance. They are defined as "teams comprised of individuals who work and live in different countries and are culturally diverse" [16]. Global teams can be used to exploit globally dispersed competencies, without the necessity to locate all the members in the same location. The possibility to locate people coming from different countries in the same place, leads to the development of global products that can satisfy the needs of different markets [16] [17]. Even if he topic of the globalization of supply chains have been studied in depth [1], a study on how supply chain management (SCM) is integrated to GPD in the global context is missing.

\section{Research Framework}

In order to fill the highlighted gaps in the literature, the main objective of the paper is to investigate how companies manage GPD activities and how they integrate such activities with SCM. To study this topic, we strive to identify common patterns in the globalization of product development activities, and those contingency factors affecting companies' behaviour in terms of GPD, such as peculiar characteristics of the firm, and location and ownership of development activities. With the aim to accomplish these research objectives we developed a research framework, and a list of research questions, that were the basis for our empirical research, as described in following sections.

In particular, the research questions we developed are the following:

- RQ1: Which are the GPD configurations adopted by companies?

- RQ2: Which is the link between the GPD configurations and the SC configurations?

- RQ3: How does contingency factors determine the choice of the GPD and the SC configurations?

- RQ4: Which is the link between GPD and the practices adopted by companies in the NPD process?

Figure 1 depicts the research framework that synthetizes the main variables and relationships expected. 


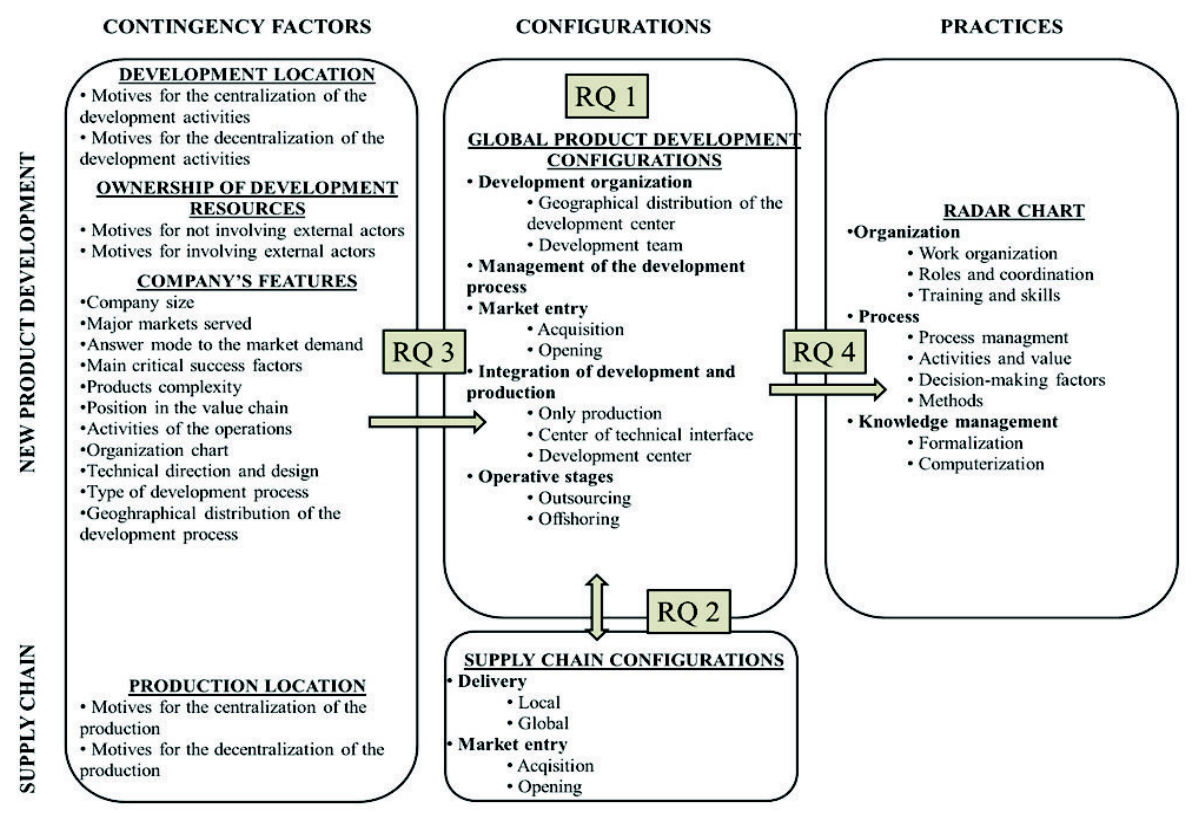

Fig. 1. Research Framework

GPD configurations describe the ways companies organize the NPD process at a global level. GPD configuration encompasses both the management of the development process [4], the market entry [18], the integration of development and production [19] [11] [13], and the operative stages [3] [20]. SC configurations represent the ways the SC is configured, thus we mainly focused on market entry [18] and the distribution of delivery, i.e. local or global.

Moreover, basing on prevalent literature contributions, we identified four elements to be the main contingency factors for GPD configurations: motives for the centralization of the development activities, motives for the decentralization of the development activities [18] [3] [19], motives for not involving external actors, motives for involving external actors [6] [19]. Additionally, two are the main contingencies we identified for the choice of SC configurations: motives for the centralization and for the decentralization of the production [21]. Moreover, company characteristics, such as company size, can affect the choice of the configuration.

Regarding the practices adopted by the companies in performing NPD process, we mainly refer to the model proposed by Rossi et al. [22], which groups NPD activities into three main areas: organization, process, and knowledge management [22].

\section{Methodology}

To explore the nature of the links between the proposed variables, we ran multiple exploratory case studies. We mainly focused on medium and big size enterprises, which are more willing to globalize their product development activities, rather than smaller firms. Moreover we decided to investigate companies' behaviour in a single sector, the electromechanical, to obtain more valuable results. The main tool used to 
gather data during the interviews has been a questionnaire, properly developed starting from literature review investigation. Aim and description of most relevant questions is summarized in Table 1.

Table 1. Questionnaire Description

\begin{tabular}{|c|c|}
\hline Topic & Description/Purpose \\
\hline NP & Indication of the success of new products developed \\
\hline SC - Make & $\begin{array}{l}\text { Level of internationalization }+ \text { reasons of relocation of production facili- } \\
\text { ties }\end{array}$ \\
\hline $\mathrm{SC}-$ Source & Inbound supply chain, procurement, location, relationship with suppliers \\
\hline SC - Delivery & Outbound supply chain \\
\hline Offshoring & Location + property of development resources \\
\hline Offshoring & Factors influencing centralization/internationalization of PD process \\
\hline Outsourcing & Role + location + stage of collaboration of actors involved in NPD \\
\hline Outsourcing & Criteria + motives of selection of development partner \\
\hline Develop. Team & Organization of PD process + internal resources + global/local team \\
\hline
\end{tabular}

The number of case studies we collected from November 2012 to January 2013 is seventeen, as displayed in Table 2.

Table 2. The sample

\begin{tabular}{c|ccc}
\hline Company & Developed Products & Empl. & Turnover [mln $\boldsymbol{~}]$ \\
\hline $\mathbf{1}$ & LED Displays & 250 & 30 \\
$\mathbf{2}$ & Electric coils, solenoids, valves & 400 & 34 \\
$\mathbf{3}$ & Forks for forklifts, lifting platform.. & 700 & 115 \\
$\mathbf{4}$ & Car electronic control & 302500 & $51,5 \mathrm{mld} €$ \\
$\mathbf{5}$ & Axles, transmissions gearboxes & 2000 & 600 \\
$\mathbf{6}$ & Payment/recognition coins; changer & 200 & 30 \\
$\mathbf{7}$ & Batteries for cars & 10300 & $2,1 \mathrm{mld} €$ \\
$\mathbf{8}$ & Motorcycles & 250 & 44 \\
$\mathbf{9}$ & Capacitors & 670 & 70 \\
$\mathbf{1 0}$ & Electronic modules, pressure sensors.. & 250 & 42 \\
$\mathbf{1 1}$ & Electric Forklifts & 5000 & $1,36 \mathrm{mld} €$ \\
$\mathbf{1 2}$ & Plugs, sockets, switch, disconnectors.. & 163 & 25 \\
$\mathbf{1 3}$ & Trucks & 336 & 60 \\
$\mathbf{1 4}$ & Industrial vacuum cleaners & 230 & 35 \\
$\mathbf{1 5}$ & Semiconductor electronic components & 50000 & $10,4 \mathrm{mld} €$ \\
$\mathbf{1 6}$ & Power transformers & 400 & 145 \\
$\mathbf{1 7}$ & Ovens, Hobs & 11500 & $1,3 \mathrm{mld} €$ \\
\hline
\end{tabular}




\section{$5 \quad$ Results Discussion}

Through the analysis of collected data we were able to answer the four identified research questions. First of all we came out with a description of possible configurations companies are adopting when performing a GPD initiative (RQ1). Main variables considered here are the geographic distribution of design sites and the location of team members, because they were better justifying and explain the differences between companies. From case studies we observed four common behaviours adopted by companies, which determine four GPD configurations, which could be named and defined as follow:

- All-in: the development is not globalized, but centralized in Italy (in one or more development canters).

- One-Gap: is the first step towards a geographically distributed development; some delocalized structures exist (center of technical interface) to customize products for local customers.

- Blind: the development is international, but each center works independently

- Connector: the development is globally distributed and the global teams collaborate for developing new product all across the world.

Different contingency factors drive the choice of companies to setup in one way rather than another $(R Q 3)$. Companies that decide to centralize development in Italy (All-In) do it to avoid the dispersion of resources, and to a lesser extent because the domestic market in which they operate is relevant. Companies that have taken the first steps in foreign territories have adopted this configuration (One-Gap) to respond quickly to customer requests. In fact, when a market becomes relevant, or when there are local needs, it may be suitable to bring the technical expertise close to the customer. Probably, when the need to integrate development and production becomes predominant, it may be reasonable to switch from having only a structure that is responsible to customize the product to a structure instead covers the whole process of development. Who decides instead to have a geographically distributed development (Blind and Connector) can globalize at least in two ways. They can acquire a foreign company with a development center able to meet the needs of the market in order to gain access to dispersed technology and competences (on a market or on a specific product family), owned by the acquired firm. Or they can integrate production with development activities, so that those who design the product are in direct contact with the production. We have observed that very often, when a foreign served market starts to become significant, the path followed by companies is to delocalize the production at first step, then the development.

Data demonstrate evidences that a link between the configurations of global product development and the supply chain exists $(R Q 2)$. We observed that similar GDP configurations have similar SC characteristics. Moreover, in term of GPD configurations and NPD adopted practices (RQ4), we noticed that All-in and One-Gap have some similarities, such as Blind and Connector. We then identified two main clusters: thin and thick. What has emerged is that companies in cluster Thin, are less structured, and for example do not have training programs for human resources nor the definition of roles and coordination are formalized process. Conversely, companies in the cluster Thick, implement tools, methods, rules, standards to guarantee highly formalized NPD process which is uniform across all corporate locations. 


\section{Conclusions}

The paper analyses in detail GPD and its link with NPD practices and SC configurations, highlighting the main contingency factors affecting the choices of organizations. Some highlights, emerging from a critical elaboration of the proposed ideas, lead us to think that in its globalization process, a company, moving from a totally centralized configuration (All-in), to a completely globalized one (Blind, Connector, One-gap), should take into account some hints:

- Changes in the process organization and in the company's culture may be required. Thus, managers have to deploy adequate resources to achieve success in the process of globalization.

- The process must be divided into clear steps, that can hence be assigned to globally dispersed development functions.

- "Do not outsource key competences" [3] is a fundamental principle of GPD.

- Members of global teams may use different technologies, systems and specific processes. Thus, it is necessary to uniform the infrastructure and the systems used.

- Team members speak different languages and have different cultural values. This may lead to misunderstandings or misinterpretations. Because of these problems, shifting to a GDP approach should follow the adoption of new modes of collaboration between globally dispersed team members.

The relocation of production does not always follow the internationalization of development: companies internationalize design with different shades, and this is according to the needs and characteristics of each company.

The conducted research, which involves seventeen companies, doesn't have statistic value but case studies serve as a mean to better understand how GPD is managed in real cases and how common paths could be found in different behaviours, within a specific context (Italian medium and big enterprises from the electromechanical sector). Future development could be the generalization of found results involving a larger number of companies, through a proper structured survey or multiple case studies. Furthermore the comparison between the highlighted results within the Italian context could be expanded to other countries and sectors.

Acknowledgements. This work was partly funded by the European Commission through the Linked Design Project (FP7-2011-NMP-ICT-FoF, http://www.linkeddesign.eu/). Part of the funds came also from the GeCo Observatory and its supporting partners. The authors wish to acknowledge their gratitude and appreciation to tall the partners and supporters for their contributions.

\section{References}

1. Cagliano, R., Caniato, F., Golini, R., Kalchschmidt, M., Spina, G.: Supply chain configurations in a global environment: A longitudinal perspective. Operations Management Research, 86-94 (2008)

2. Pero, M., Abdelkafi, N., Sianesi, A., Blecker, T.: A Framework for the Alignment of New Product Development and Supply Chains. Supply Chain Management: An International Journal 15(2), 115-128 (2010) 
3. Eppinger, S.D., Chitkara, A.R.: The Practice of Global Product Development (With Updates by Steven D. Eppinger). MIT Sloan Management Review (2009)

4. Ulrich, K.T., Eppinger, S.D.: Progettazione e sviluppo prodotto. McGraw-Hill, New York (2007)

5. Tidd, J., Bodley, K.: The influence of project novelty on the new product development process. R\&D Management (2002)

6. Griffith, Harmancioglu, Droge: Governance decisions for the offshore outsourcing of new product development in technology intensive markets (2009)

7. Zhao, Y., Calantone, R.: The trend toward outsourcing in new product development: case studies in six firms. International Journal of Innovation Management 7, 51-66 (2003)

8. Kahn, K.B., McDonough III, E.F.: An Empirical Study of the Relationships among Colocation, Integration, Performance, and Satisfaction. Journal of Product Innovation Management 14(3), 161-178 (1997)

9. Gomes, P.J., Joglekar, N.R.: Linking modularity with problem solving and coordination efforts. Managerial and Decision Economics 29, 443-457 (2008)

10. Albizzati, F., Pero, M., Sianesi, A.: Integrating activities and resources in global product development. In: Proceedings of the 17th International Working Seminar on Production Economics, Innsbruck, Austria, February 20-24, vol. 4, pp. 1-12 (2012)

11. von Zedtwitz, M., Gassmann, O.: Market versus technology drive in R\&D internationalization: four different patterns of managing research and development. Research Policy, 569-588 (2002)

12. Galina, S.R., Plonsky, G.A.: Global product development in the telecommunication industry: an analysis of the brazilian subsidiaries involvement. In: Proceedings of the 9th International Product Development Conference, European Institute for Advanced Studies in Management (EIASM), Sophia-Antipolis, France (2002)

13. Kumar, N.: Determinants of location of overseas R\&D activity of multinational enterprises: the case of US and Japanese corporations. Research Policy, 159-174 (2001)

14. Gassmann, O., Von Zedtwitz, M.: Trends and determinants of managing virtual R\&D teams. R\&D Management (2003)

15. PTC, PTC white paper: Gaining competitive advantage through global product development, http: / /www.ptc.com

16. McDonough, Kahn, Barczak: An Investigation of the Use of Global, Virtual, and Colocated New Product Development Teams (2001)

17. Boutellier, R., Gassmann, O., Macho, H., Roux, M.: Management of Dispersed Product Development Teams: The Role of Information Technologies. R\&D Management 28, 13-25 (1998)

18. Terzi, S., Garetti, M.: Global Product Development in Italian companies: an empirical analysis (2012)

19. Ulrich, K.T., Ellison, D.J.: Beyond make-Buy: Internalization and Integration of Design and Production. Production and Operations Management, 1-32 (2005)

20. Quinn, J.B.: Outsourcing innovation - the new engine of growth. Sloan Management Review 41, 13-28 (2000)

21. Faraone: La delocalizzazione produttiva come attività generatrice di valore (2009)

22. Rossi, M., Terzi, S., Garetti, M.: Proposal of an Assessment Model for New Product Development. In: Emmanouilidis, C., Taisch, M., Kiritsis, D. (eds.) Advances in Production Management Systems. IFIP AICT, vol. 397, pp. 383-390. Springer, Heidelberg (2013) 